\title{
Facilitação neuromuscular proprioceptiva na dermatomiosite juvenil: relato de caso
}

\author{
Neuromuscular facilitation in proprioceptive \\ juvenile dermatomyositis: a case report
}

\author{
Egberto Luiz Felício Junior ${ }^{1}$, Gustavo Tinti ${ }^{1}$, Marielza R. Ismael \\ Martins $^{2}$, Marcos Henrique D’Aglio Foss ${ }^{3}$, Márcia Taves Parisi ${ }^{3}$
}

Felicio Junior EL, Tinti G, Martins MRI, Foss MHD, Parisi MT. Facilitação neuromuscular proprioceptiva na dermatomiosite juvenil: relato de caso. Rev Med (São Paulo). 2009 jul.dez.;88(3/4):186-90.

RESUMO: INTRODUÇÃO: Dermatomiosite Juvenil (DMJ) é uma doença muscular inflamatória de etiologia desconhecida, auto-imune, e que se manifesta nos pequenos vasos sanguíneos dos músculos e pele. A incidência varia entre 0,8 e 4,1 por milhão de crianças nascidas por ano, com prevalência do sexo feminino. São cinco os critérios de diagnóstico: fraqueza simétrica proximal progressiva, enzimas musculares elevadas, anormalidades no eletromiograma, padrão inflamatório na amostra da biópsia muscular, e associação com distúrbios cutâneos. OBJETIVO: Relatar a Facilitação Neuromuscular Proprioceptiva (FNP) em um caso de DMJ. MÉTODO: Foi avaliada uma paciente do sexo feminino, nove anos de idade, com quadro de DMJ e com sintomas de: fraqueza muscular, dificuldade em atividades de vida diária e quedas freqüentes, que se encontra em acompanhamento médico ambulatorial no Hospital Foram realizadas três avaliações, antes (AV-1), durante (AV-2) e após (AV-3) a intervenção fisioterapêutica. Após a primeira avaliação, foi aplicado um tratamento com 24 sessões, com duração de uma hora cada sessão, durante 12 semanas, que constou de Facilitação Neuromuscular Proprioceptiva (FNP) com técnicas de estímulo de estiramento e reversões lentas nas diagonais primitiva e funcional, bem como alongamentos associados. RESULTADOS: Aumento da força muscular em membros inferiores, diminuição de contraturas, ganho de amplitude de movimento articular e melhor qualidade de vida para a paciente. CONCLUSÃO: Em vista dos resultados expostos, a utilização da FNP foi satisfatória, e estudos longitudinais são necessários para que se comprove a eficácia a longo prazo.

DESCRITORES: Propriocepção; Dermatomiosite; Doenças musculares; Doenças auto-imunes; Fisioterapia.

\footnotetext{
1. Aprimorandos de Fisioterapia, Bolsistas Fundap, Hospital de Base, Faculdade de Medicina de São José do Rio Preto FAMERP.

2. Professora Doutora, Terapeuta Ocupacional, Departamento de Ciências Neurológicas - FAMERP.

3. Professores, Fisioterapeutas, Departamento de Ciências Neurológicas - FAMERP.

Endereço para correspondência: Depto. de Ciências Neurológicas - FAMERP. Av. Brigadeiro Faria Lima, 5416 - Vila São Pedro - CEP: 150090-000 - São José do Rio Preto, SP.
} 


\section{INTRODUÇÃO}

$\mathrm{D}$ ermatomiosite Juvenil (DMJ) é uma doença muscular inflamatória de etiologia desconhecida, considerandose a hipótese auto-imune, e que se manifesta nos pequenos vasos sanguíneos dos músculos e pele ${ }^{1}$. Segundo Callen e Wortmann², fatores de interação ambientais e infecções em um indivíduo com predisposição imunogenética são coadjuvantes no desenvolvimento da doença na forma infantil.

Clinicamente, é caracterizada por: fraqueza muscular, fadiga, dor muscular e articular ${ }^{1}$. A descamação da pele tipicamente localizada próxima às articulações (pápulas de Gottron), é encontrada com frequência ${ }^{3}$,além de vermelhidão nas faces com algum edema em volta dos olhos (eritema peri-orbital) e nas bochechas (rash malar).

Embora o processo comprometa primeiramente a pele e os músculos proximais, podendo levar a depósitos de cálcio dentro dos tecidos com perda da função muscular e limitações graves, é uma doença sistêmica com manifestações freqüentes no trato gastrointestinal e no sistema cardiopulmonar ${ }^{4}$.

A presença de dermatite seborréica, psoríase e alopécia pode estar relacionada à doença ${ }^{5}$.

Sua incidência varia entre 0,8 e 4,1 por milhão de crianças nascidas por ano, com prevalência no sexo feminino ${ }^{3}$.

De acordo com Callen e Wotmann², são cinco os critérios de diagnósticos, como segue: fraqueza simétrica proximal progressiva, enzimas musculares elevadas (principalmente a creatinofosfoquinase CPK), anormalidades no eletromiograma, padrão inflamatório na amostra da biópsia muscular e associação com distúrbios cutâneos.

Tratamentos convencionais incluem: corticosteróides, metotrexato, ciclosporina, imunoglobulina intravenosa, agentes biológicos, como uma importante citocina com efeito anti-TNF (ainda em fase experimental), e de terapia tópica com uma equipe multiprofissional (fisioterapeutas, terapeutas ocupacionais e psicólogos) ${ }^{6}$.

Os objetivos do tratamento incluem controlar a doença e prevenir as complicações, tais como: calcificações, contraturas e osteoporose, que é comumente secundária à toxicidade das medicações $^{7}$.

Diversos estudos demonstram a segurança na realização de programas de exercícios de leve a moderada intensidade a fim de reduzir a inabilidade dos pacientes com $\mathrm{DMJ}^{8,9}$. De acordo com estudos recentes, os exercícios resistidos são recomendados na reabilitação desses pacientes ${ }^{10,11}$.

Evidências mostram que os exercícios concêntricos e excêntricos são os programas de treinamento mais eficazes para todas as idades, porém a literatura nacional tem poucos estudos nesse sentido ${ }^{7,10}$.

Um modelo de programa de exercício programado por um fisioterapeuta especialista em reumatologia pediátrica incluía exercícios isotônicos concêntricos e excêntricos em grupos musculares específicos (região anterior, posterior, medial e lateral dos membros inferiores), realizado em cadeia cinética fechada ${ }^{4}$.

Alguns estudos investigaram os efeitos do exercício terapêutico nos pacientes com dermatomiosite. Esses estudos mostraram que exercícios resistidos e não-resistidos melhoram a oferta de oxigênio, força e função muscular ${ }^{12,13}$.

Outros estudos relatam que a corticoterapia e terapias imunossupressoras, quando associadas à fisioterapia, mostram melhores resultados ${ }^{2,11}$.

Nesse contexto, o objetivo deste relato é ilustrar o trabalho fisioterapêutico utilizando a abordagem de facilitação neuromuscular proprioceptiva em um caso de dermatomiosite juvenil do Ambulatório.

\section{MÉTODO}

\section{Caso}

Criança, sexo feminino, nove anos de idade, branca, foi encaminhada para o serviço de fisioterapia com diagnóstico de dermatomiosite juvenil, após biópsia muscular e eletroneuromiografia (agosto/2005). Apresentava quedas frequentes há mais de quatro anos e piora progressiva do quadro, bem como déficits em atividades de vida diária (AVDs) tais como vestir-se, e em atividades motoras, tais como agachar-se, correr, andar de bicicleta e subir escadas.

\section{Manifestações clínicas}

Apresentou diminuição da força muscular em: (1) flexores/extensores do quadril D./E.: grau III; (2) abdutores do quadril D./E.: grau III; (3) flexores/ extensores do joelho D./E.: grau IV; (4) flexores 
plantares/dorsiflexores do pé D./E.: grau IV).

$\mathrm{Na}$ biopsia muscular e eletroneuromiografia, os achados foram: variações do calibre das fibras musculares, com presença de fibras fendidas, núcleos internos, fibras em degeneração (necrose) e fibras em regeneração (fibras basofílicas). Foi identificada presença de evidente atrofia perifascicular.

Notou-se também perda da atividade oxidativa nas fibras distribuídas na periferia dos fascículos. Também se evidenciou discreta proliferação do tecido conjuntivo endomisial e perimisial. Observou-se infiltrado inflamatório linfomononuclear endomisial, perimisial e perivascular.

Diversas fibras musculares e vasos apresentaram positividade à reação pela fosfatase ácida.

\section{Avaliação fisioterápica}

$\mathrm{Na}$ avaliação inicial, ao exame físico, apresentou limitações em amplitude de movimento, por encurtamentos (calcificações) musculares, das seguintes articulações: coxofemoral (flexão do quadril D.: 115\%/ E.: 110\%/ abdução do quadril D./E.: 30%), fêmoro-tibial (flexão do joelho D./E.: 115º), tíbio-talar (flexão plantar D./E.: 45을 e dorsiflexão D./E.: 5º). Para a realização de tais mensurações, utilizou-se o goniômetro da marca Carci. Ainda, identificaram-se: rash malar bilateral, semidependência em AVDs, marcha sem dissociação de cinturas escapular e pélvica, além de fase de apoio de calcanhar com borda externa e sem apoio de hálux. Observou-se um estado emocional negativo e dor à movimentação articular, verificada através da Escala analógica $(8)^{14}$. No momento, em uso de acido fólico (5mg,), prednisona $(5 \mathrm{mg} / \mathrm{d})$, metotrexato $(25 \mathrm{mg})$ e carbonato de cálcio $(500 \mathrm{mg} / \mathrm{d})$.

\section{Procedimento fisioterápico}

O tratamento foi composto por 12 sessões de fisioterapia, durante seis semanas, com duração de uma hora cada sessão, tendo sido reavaliada três vezes com intervalo de duas semanas.

A terapia utilizou o método da facilitação neuromuscular proprioceptiva (FNP) com técnicas de estímulo de estiramento e reversões lentas, nas diagonais primitivas e funcionais, além de variantes, composta por duas séries de 15 repetições cada, associadas a alongamentos dos músculos encurtados: quadríceps, isquiotibiais, glúteos, adutores, tibial anterior, tríceps sural.

\section{RESULTADOS}

Na segunda avaliação, foram observadas as seguintes amplitudes de movimento nas articulações: coxofemoral (flexão do quadril D.: 120\% E.: 115\%), fêmoro-tibial (flexão do joelho D./E.: 125ํ), tíbiotalar (flexão plantar D./E.: 60ำ e dorsiflexão D./E.: $\left.10^{\circ}\right)$. Apresentou ao hemograma um CPK de (128 U/L), rash malar bilateral, melhora da força muscular em: (1) flexores/extensores do quadril D./E.: grau III; (2) abdutores do quadril D./E.: grau IV, (3)flexores/extensores do joelho D./E.: grau IV, (4)flexores plantares do pé D./E.: grau IV/ dorsiflexores do pé D./E.: grau V), manobra deficitária de Mingazzini não realizada, melhora de AVDs (conseguiu agachar, trocar vestuários; diminuição das quedas), e importante melhora da marcha, agora com dissociação de cinturas escapular e pélvica, normalidade nas fases de apoio. Observou-se um estado emocional positivo da paciente e diminuição da dor à movimentação articular nos alongamentos (escala analógica: 5).

$\mathrm{Na}$ avaliação final, sob permanência da corticoterapia nas mesmas doses, obtiveram-se os seguintes resultados na amplitude de movimento articular: coxofemoral (flexão do quadril D.: 120\%/ E.: 120\%/), fêmoro-tibial (flexão do joelho D./E.: 125으, tíbio-talar (flexão plantar D./E.: 60ำ e dorsiflexão D./E.: 15ㅇ).

Houve diminuição do rash malar bilateral e melhora da força muscular em: (1)flexores/extensores do quadril D./E.: grau IV, (2)abdutores do quadril D./E.: grau V, (3)flexores/extensores do joelho D./E.: grau V, (4)flexores plantares do pé D./E.: grau V/ dorsiflexores do pé D./E.: grau V).

Melhorou a independência em AVDs, conseguiu agachar-se, trocar vestuário, correr, subir degraus, além de ausências de quedas (realizou o trajeto para a escola sozinha) e marcha normal. Observou-se uma melhora significativa do estado emocional da paciente e diminuição da dor aos alongamentos.

\section{DISCUSSÃO}

Dermatomiosite juvenil (DMJ) é uma doença reconhecida e rara, inserida na classe das vasculapatias pediátricas. Caracteriza-se clinicamente 
Felício Junior ELF, et al. Facilitação neuromuscular proprioceptiva na dermatomiosite.

por fraqueza muscular progressiva, inicialmente nos músculos proximais, com expansão distal. A doença pode ser sistêmica, podendo afetar vários órgãos e causar graves morbidades ${ }^{4}$.

A calcinose ou calcificação de tecidos não-articulares é uma complicação freqüente da dermatomiosite juvenil (DMJ), com incidência entre $10 \%$ e $70 \%$. Pode ser dolorosa e muito debilitante, resultando, às vezes, em incapacidade funcional. A patogênese é desconhecida, mas parece envolver a ação de células inflamatórias, citocinas e proteínas da matriz mineralizada, como osteocalcina. Atraso no início do tratamento, vasculopatia e doença grave são fatores de risco para o aparecimento de calcinose nesses pacientes ${ }^{15}$.

Os depósitos de cálcio podem aparecer no tecido subcutâneo, na fáscia muscular ou intramuscular, muitas vezes impedindo os movimentos ${ }^{16}$.

Em nosso estudo, a paciente apresentou calcificações, dado concordante com outros trabalhos nos quais a frequencia de calcificações é alta ${ }^{17,18}$.

A paciente descrita apresentou limitações importantes em AVD'S devido encurtamentos, fraquezas musculares e quadro álgico à movimentação articular, caracteristicas próprias desta afecção ${ }^{15,19}$.

Stringer e Feldman ${ }^{7}$ descrevem que a fisioterapia desempenha um papel importante na reabilitação de pacientes com DMJ com fraqueza muscular e limitações em AVDs, corroborando Cleland e Venzke ${ }^{12}$ que refere que a fisioterapia na DMJ visa a estabelecer a independência com um programa de exercício que maximize a força e função muscular através de exercícios concêntricos e excêntricos.

Vários estudos comprovam a eficácia de exercícios concêntricos e excêntricos realizados como forma de tratamento para incrementar a força muscular e diminuir possíveis calcificações musculares, bem como propiciar ganho de amplitude de movimento articular, de equilíbrio e de coordenação ${ }^{1,4}$.

A paciente relatada faz uso de corticóides e, devido à existência de várias alternativas fisioterapêuticas para a realização de exercícios isotônicos, esse estudo compactua do uso do método da FNP como forma de tratamento, além de alongamentos associados.

O método da FNP foi inicialmente desenvolvido no ano de 1940 e início dos anos 50 por Kabat et al., como uma modalidade de tratamento para as paralisias. Originalmente concebida para facilitar a flexibilidade, força e coordenação, a FNP se tornou um recurso terapêutico eficaz e de freqüente utilização ${ }^{20}$.

AFNP é utilizada para melhorar o desempenho e a performance física de atletas, sedentários saudáveis ou portadores de disfunções orgânicas, e principalmente em recuperação neuromuscular. Estudos que aplicam exercícios com as técnicas de FNP em outros vários segmentos corporais possibilitam treinar o indivíduo com uma combinação de eixos articulares, tornando o movimento mais próximo daquele que é exigido nas atividades de vida diária ${ }^{21}$.

No estudo de Gama e colaboradores ${ }^{22}$, o terapeuta, ao realizar o alongamento dos músculos isquiotibais, flexionava passivamente o quadril da paciente até o limiar de dor, posição em que era referido desconforto nos isquiotibiais. Ao sinal do pesquisador, era solicitado que a paciente realizasse força máxima para estender a perna, contraindo os extensores do quadril por cinco segundos; a contração era resistida pelo terapeuta. Ao final dos cinco segundos, a paciente relaxava a musculatura e logo em seguida tinha o quadril flexionado passivamente, com o joelho estendido, até referir novamente desconforto. O membro era mantido nesse ponto por 30 segundos. As pacientes foram instruídas a relaxar totalmente a perna alongada durante o alongamento, não oferecendo nenhuma resistência.

No manejo da paciente referida, utilizou-se essa técnica de alongamento para todas as cadeias musculares que apresentaram encurtamentos, acrescido de apresentação da escala analógica da dor ao final de cada sessão, devido a quadro álgico à movimentação articular no período dos alongamentos. Conforme avaliado nos resultados, houve melhora da amplitude de movimento articular, da força muscular, das atividades da vida diária, além de diminuição progressiva da dor à movimentação articular.

\section{CONCLUSÃO}

A associação do método da facilitação neuromuscular proprioceptiva como tratamento na dermatomiosite juvenil é raro na literatura nacional. Neste estudo, sua utilização foi satisfatória, pois houve aumento da força muscular, melhora significativa das AVDs, aumento da amplitude de movimento articular e consequentemente melhor qualidade de vida para a paciente.

Mesmo tendo sido obtidos bons resultados, estudos longitudinais são necessários para que se comprove uma eficácia apurada. 
Felicio Junior EL, Tinti G, Martins MRI, Foss MHD, Parisi MT. Neuromuscular facilitation in proprioceptive juvenile dermatomyositis: a case report. Rev Med (São Paulo). 2009 jul.dez.;88(3/4):186-90.

ABSTRACT: INTRODUCTION: Juvenile dermatomyositis (DM) is an inflammatory muscle disease of unknown etiology, autoimmune disorder, which manifests itself in small blood vessels of the muscles and skin. The incidence varies between 0.8 and 4.1 per million children born each year with a prevalence of females. Five criteria are considered for diagnosis: progressive proximal symmetrical weakness, elevated muscle enzymes, abnormal electromyogram and inflammatory pattern in the muscle biopsy sample, and association with cutaneous disorders. OBJECTIVE: To report the Neuromuscular Facilitation Proprioceptive (PFN) procedure in a case of juvenile DM. METHODS: We evaluated a female patient, nine years old, with part of JDM, who manifested: muscle weakness, difficulty in daily activities and frequent falls. She attended the out patient service at our Hospital Three evaluations were performed before (AV-1), during (AV-2) and after (AV-3) to physiotherapy intervention. After the first evaluation, a treatment was applied in 24 sessions over 12 weeks, lasting an hour each, which consisted of Neuromuscular Facilitation proprioceptive (FNP) with stimulating techniques of stretching and slow reversals in diagonal primitive and functional as well as associated stretching. RESULTS: Increased muscle strength in lower limbs, reduction of contractures, gain range of motion and better quality of life for the patient. CONCLUSION: Considering the above results, longitudinal studies are required to prove efficacy in long-term.

KEY WORDS: Proprioception; Dermatomyositis; Muscular diseases; Autoimmune diseases; Physiotherapy.

\section{REFERÊNCIAS}

1. Alexanderson $\mathrm{H}$, Dastmalchi M, Liljedahl ME, Opava $\mathrm{CH}$, Lundberg IE. Benefits of intensive resistance training in patients with chronic polymyositis or dermatomyositis. Arthritis Rheum. 2007;57:768-77

2. Callen JP, Wormann RL. Dermatomyosits. Clin Dermatol. 2006;24:363-73.

3. McCann LJ, Juggins AD, Maillard SM, Wedderburn LR, Davidson JE, Murray KJ, Pilkington CA. The Juvenile Dermatomyositis National Registry and Repository (UK and Ireland) - clinical characteristics of children recruited within the first $5 \mathrm{yr}$. Rheumatology. 2006;45:1255-60.

4. Maillard SM, Jones R, Owens CM, Pilkington C, Woo PM, Wedderburn LR, Murray KJ. Quantitative assessments of the effects of a single exercise session on muscles in juvenile dermatomyositis. Arthritis Rheum. 2005;53:558-64.

5. Kasteler JS, Callen JP. Scalp involvement in dermatomyositis: often overlooked or misdiagnosed. JAMA. 1994;272:1939-41.

6. Kim S, El-Hallak M, Dedeoglu F, Zurakowski D, Fuhlbrigge $\mathrm{RC}$, Sundel RP. Complete and sustained remission of juvenile dermatomyositis resulting from aggressive treatment. Arthritis Rheum. 2009;60:1825-30.

7. Stringer E, Feldman BM. Advances in the treatment of juvenile dermatomyositis. Curr Opin Rheumatol. 2006;18:503-6.

8. Shiari R, Sara E, Atoosa G, Farzaneh J. Amyopathic juvenile dermatomyositis: a case presentation. Joint Bone Spine. 2009 May 20.

9. Iorizzo LJ $3 R D$, Jorizzo JL The treatment and prognosis of dermatomyositis: na updated review. J Am Acad Dermatol. 2008;59:99-112.

10. Stringer E, Feldman BM. Advances in the treatment of juvenile dermatomyositis. Curr Opin Rheumatol. 2006;18:503-6.

11.Alexanderson $\mathrm{H}$. Exercise: an important component of treatment in the idiopathic inflammatory myopathies.Curr Rheumatol Rep. 2005;7:115-24.

12. Cleland JA, Venzke JW. Dermatomyosits: evolution of a diagnosis. Phys Ther. 2003;।.83:932-45.
13. Alexanderson $\mathrm{H}$, Lundberg IE. The role of exercise in the rehabilitation of idiopathic inflammatory myopathies. Curr Opin Rheumatol. 2005;17:164-71.

14. Piovesan EJ, Lange MC, Kowacs PA, Pacheco C, Werneck LC. Evaluation of headache intensity in migrainous patients with visual handicap through the tactile analogical scale (TAS). Arq Neuropsiquiatr. 2001;59:702-7.

15. Castro TCM, Yamashita E, Terreri MT, Len CA, Hilário MOE. Calcinose na infância, um desafio terapêutico. Rev Bras Reumatol. 2007;47:10-5.

16. Romero KT, Terreri MT, Len CA, Hilário MO. Dermatomiosite e polimiosite juvenis: diagnóstico e tratamento. Rev Paul Pediatria. 2003;21:223-7.

17. Scola RH, Werneck LC, Prevenello DMS, Toderke EL, Iwamoto FM. Diagnosis of dermatomyositis and polymyositis: a study of 102 cases. Arq Neuropsiquiatr. 2000;58:789-99.

18. Sallum AME. Expressão das moléculas de adesão (ICAM-1 e VCAM-1) em tecido muscular de pacientes com dermatomiosite juvenil [Dissertação]. São Paulo: Faculdade de Medicina, Universidade de São Paulo; 2002.

19. Martini G, Calabrese F, Biscaro F, Zulian F. A child with ermatomyositis and a suspicious lymphadenopathy. J Rheumatol. 2005;32:744-6.

20. Burke DG, Culligan LE. The theorical basis of proprioceptive neuromuscular facilitation. J Strength Cond Res. 2000;14:496500.

21. Moreno MA, Silva E, Gonçalves M. O efeito das técnicas de facilitação neuromuscular proprioceptiva- método Kabat- nas pressões respiratórias máximas. Fisioter Mov (Curitiba). 2005;18: 53-61.

22. Gama ZA da S, Medeiros CA de S, Dantas AVR, Souza TO. Influência da freqüência de alongamento utilizando facilitação neuromuscular proprioceptiva na flexibilidade dos músculos isquiotibiais. Rev Bras Med Esporte. 2007;13:33-8.

Recebido para publicação em: 23/06/2009 Aceito para publicação em: 12/11/2009 